\title{
Erwartungsbildung und Prognose Ergebnisse einer experimentellen Studie
}

\author{
Otwin Becker ${ }^{1}$ und Ulrike Leopold-Wildburger ${ }^{2}$ \\ ${ }^{1}$ Alfred Weber Institut, Ruprecht Karls-Universität Heidelberg \\ ${ }^{2}$ Institut für Statistik und OR, Karl Franzens-Universität Graz
}

\author{
„Das Auge war von jeher weitsichtiger als alle anderen Sinne" \\ Loblied und andere Olivenkerne, Rafik SCHAMI, 1997
}

\begin{abstract}
Zusammenfassung: Die visuell gestützte Extrapolation ist ein besonders häufig angewendetes Verfahren, das praktisch für jede graphisch dargestellte Zeitreihe Verwendung finden kann. Folgt die Zeitreihe keinem leicht erkennbaren, strengen Bildungsgesetz, weichen die individuellen Gestaltsausdeutungen in der Regel sehr voneinander ab, jedoch im Durchschnitt mitteln sich singulär viele Ausdeutungen heraus. Demgegenüber lassen sich einige wenige wesentliche Merkmale ausfindig machen, die im vorliegenden Beitrag die Grundlage eines apriori Erklärungsmodells für Kollektivprognosen bilden. Die Überprüfung dieses Modells im Rahmen einer Reihe von Experimenten liefert bemerkenswerte Ergebnisse.
\end{abstract}

\begin{abstract}
Among qualitative forecasting techniques, graphic extrapolation of a time series is a particularly simple and by far the most popular forecasting technique. Recognising regularities of a given curve by visual inference enables the filtering out of outliers and often also helps to identify clearly the patterns of the stochastically superimposed formation rules according to which a certain series of numbers is formed. This survey presents an a priori model for explaining average forecasts which have already furnished remarkable results in a number of experimental verification runs.
\end{abstract}

Schlüsselwörter: Prognosen, Erwartungsbildung, Extrapolation,Visuelle Inferenz, Graphische Zeitreihenanalyse, Experimente.

\section{Einführung}

Die in diesem Aufsatz vorgegebene Problemstellung zur Erwartungsbildung behandelt eine Thematik, die in der wirtschaftswissenschaftlichen Forschung weit zurückreicht. Die Erwartungen von Entscheidungsträgern haben in der ökonomischen Verhaltensforschung von jeher als antizipierte Größen, mit denen man rechnen muß, eine wesentliche Rolle gespielt. Die Berücksichtigung solcher Einzelfaktoren zur Erklärung wirtschaftlicher Entscheidungen läßt sich bis in die Anfangsjahre des vergangenen Jahrhunderts zurückverfolgen. So behandelt etwa der als Vertreter der Banking-Schule bekannte Henry Thorton (1802) den Einfluß der Preiserwartungen auf Zinssätze. 
Von den Vertretern der Stockholmer Schule wurde in den dreißiger Jahren dieses Jahrhunderts systematisch untersucht, wie Abweichungen zwischen geplanter (bzw. erwarteter) und tatsächlicher Entwicklung den Wirtschaftsablauf beeinflussen. Richtungsweisend hierzu waren vor allem die Arbeiten von Erik Lindahl (1939).

Aus dem Kreis der älteren Nationalökonomen ist vor allem J.M. Keynes zu nennen, der die Erwartungen der Wirtschaftssubjekte der Unternehmer (Investoren), Sparer und Verbraucher in den Mittelpunkt der theoretischen Deutung des Wirtschaftsprozesses rückte. Obgleich Keynes selbst auch noch in seiner „General Theory" Erwartungen als Daten - in modelltheoretischer Sprechweise genauer als exogene Größen - verwendete, hat er doch entscheidende Anstöße für die weitere Forschung gegeben.

Von den Vertretern der „New Economics“ war es vor allem Hicks (1939), der mit der Konzeption der „Elasticity of Expectations“ auf eine Endogenisierung der Erwartungsgrößen hinwirkte. Der von Hicks unterstellte Zusammenhang läuft auf eine adaptive Erwartungsbildungsformel hinaus, die den Prozeß der Erwartungsbildung als solchen jedoch nur unzureichend erklärt. Im selben Sinne ist auch die Forderung von Schumpeter (1954) zu verstehen, der in seinen „Business Cycles“ schreibt: „Unless we know why people expect what they expect, any argument is completely valueless which appeals to them as causae efficientes". Und an früherer Stelle derselben Schrift: „If we discontinue the practice of treating expectations as if they were ultimate data, and treat them as what they are - variables which it is our task to explain - properly linking them up with the business situations that give rise to them, we shall succeed in restricting expectations to those which we actually observe and not only reduce their influence to its proper proportions but also understand how the course of events molds them and at certain times so turns them as to make them work toward equilibrium".

Es gibt sowohl theoretische, meist mikrofundierte, als auch empirische Studien zur Erwartungsbildung (Murphy, 1997, Ladreiter und Leopold, 1999). Weniger bekannt sind experimentelle Arbeiten zur Erwartungsbildung, wobei im folgenden über eine davon berichtet werden soll.

Die Methode des kontrollierten Experiments, die sich ja in anderen Wissenschaften, zum Beispiel in der Psychologie, so hervorragend bewährt hat, hat für die Analyse der Erwartungsbildung eine Reihe von Vorteilen. Im Experiment gelingt es nämlich, das Wissen der Versuchspersonen und die ihnen zugehenden Informationen zu kontrollieren, systematisch zu verändern und das Experiment beliebig oft zu wiederholen.

Die beliebige Wiederholbarkeit des Experiments erlaubt es, allgemeine Aussagen sowohl über Individualprognosen, als auch aus deren Durchschnitt Aussagen über die Kollektivprognosen (average opinions) zu analysieren.

Diesbezüglich hat das experimentelle Vorgehen eindeutige Vorteile gegenüber empirischen Untersuchungen, wie z.B bei Befragungen es sich nicht ausschließen läßt, daß die einzelnen Befragten von unterschiedlichem Vorwissen ausgehen und ggf. noch unterschiedliche Prognoseziele verfolgen. Dieser Vorteil erklärt das Expandieren des Wissenschaftszweiges der experimentellen Wirtschaftsforschung, experimental economics, neuerdings auch behavioral economics genannt. 
Die typische Vorgehensweise in der experimentellen Wirtschaftsforschung besteht darin, Experimente immer auf der Grundlage vereinfachter Referenzsituationen aufzubauen. Die dabei gefundenen Ergebnisse beschreiben zunächst noch nicht das gesamte informationsverarbeitende „Programm“, sondern allenfalls einzelne „Prozeduren" daraus. Es besteht jedoch Grund zu der Annahme, daß auch die Prognosen in komplexeren Referenzsituationen durch dieselben Erwartungsbildungsregeln erklärt werden können.

In vielen Fällen aus naturwissenschaftlichen und technischen, sowie wirtschaftswissenschaftlichen Bereichen steht der historische Verlauf einer Zeitreihe bis zur Gegenwart als Abbildung zur Verfügung. Es interessiert dann, eine visuell gestützte Aussage über den weiteren Verlauf der Zeitreihe machen zu können. In dem vorliegenden Experiment liegt jeder Versuchsperson eine Zeitreihe in graphischer Darstellung als Chart vor. Es wird dann die Aufgabe gestellt, unter der Bedingung rein visueller Verarbeitung der Information, die Kurve über den Endpunkt hinaus als Prognose zu verlängern. Das Hauptziel der vorliegenden Arbeit ist es, die Vorhersagen der Versuchspersonen (Vpn), primär die kollektive Erwartungsbildung, unter Berücksichtigung der jeweils verfügbaren Information zu erklären. Ein Modell, das hierzu entwickelt wurde, ist die sogenannte bounds \& likelihood-Prozedur.

Die vorgestellte bounds \& likelihood-Prozedur erklärt die kollektiven Prognosen fast ebenso gut, wie dies mit den rationalen Erwartungswerten im Sinne von John F. Muth (1961) möglich ist. Der Rational-Expectations-Hypothesis-Ansatz läßt sich wie folgt definieren: $f_{t+1}=E\left[x_{t+1} \mid \Omega_{t}\right]$, wobei die mathemathische Erklärung in dieser Gleichung im allgemeinen eine Funktion aller zum Zeitpunkt t verfügbaren Systemvariablen ist und die Kenntnis des (wahren) Systemmodells vorausgesetzt wird.

Dabei interessiert uns auch darüber hinaus die Frage, inwieweit das Erklärungsmodell als eine effiziente Heuristik für die Vorhersagen ähnlicher stochastischer Folgen angewendet werden kann. (Vgl. Becker und Leopold-Wildburger, 1996.)

\section{Das Experiment}

Das Experiment wurde mittels der in Abbildung 1 wiedergegebenen Zeitreihe durchgeführt. Die Versuchspersonen (Vpn) mußten in jeder Periode $t(t=0, \ldots, 41)$ möglichst gut den Wert für die nächstfolgende Periode vorhersagen. Sie sahen hierzu die Zeitreihe bis zur Periode $t$ und erfuhren nach ihrer Prognose den tatsächlichen Wert der Zeitreihe. Die im Experiment verwendete Zeitreihe wurde erzeugt mittels der Differenzengleichung

$$
x_{t}=a_{0}+a_{1} x_{t-1}+a_{2} x_{t-2}+u_{t} \quad \text { für } t=1,2, \ldots .
$$

Die Differenzengleichung (1) ist ein AR(2)-Prozeß, in dem als Koeffizienten $a_{0}=3.5$, $a_{1}=1$ und $a_{2}=-0.5$ gewählt wurden. Die zufällige Störgröße $\left(u_{t}\right)$ des Systems ist gleichverteilt im Intervall $(-3 ; 3)$. Zur Vereinfachung des Experiments wurden die Zahlen jeweils auf ganze Einheiten gerundet. Die der Gleichung (1) zugeordnete homogene Differenzengleichung erzeugt für diese Parameterkombination gedämpfte Schwingungen. Die Kurvengestalt der Zeitreihe ähnelt geringfügig verrauschten 
Konjunkturschwankungen, wie sie in der ökonomischen Realität häufig vorzufinden sind.

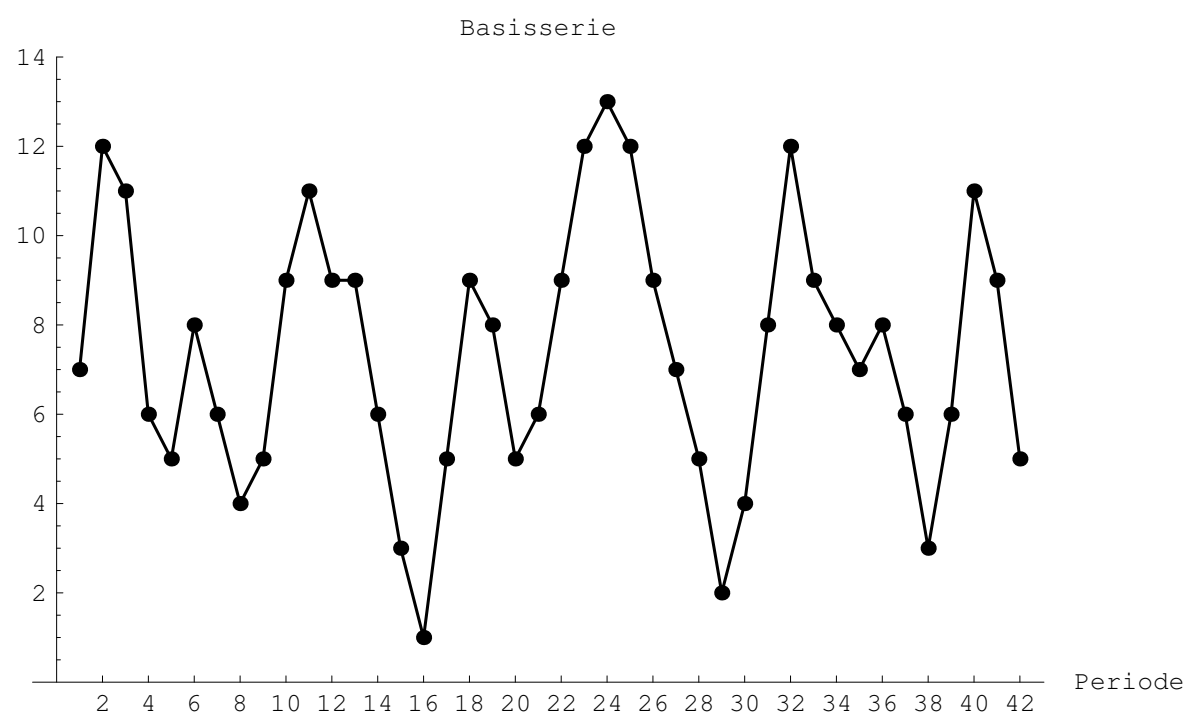

Abbildung 1: Der Verlauf der Zeitreihe $x_{t}$.

Die Vpn wurden über die Bedeutung der Reihe nicht aufgeklärt; ihre Information bestand ausschließlich aus den bis dahin jeweils sichtbaren Punkten der Zeitreihe.

Die rationalen Erwartungen zur Differenzengleichung (1) ergeben sich, indem die Zufallsvariable $\mathrm{u}_{t}$ durch ihren Erwartungswert $E\left(u_{t}\right)=0$ ersetzt wird. Da die Vpn weder die Differenzengleichung noch die Verteilung der Zufallsvariablen kannten, waren sie natürlich nicht in der Lage, die rationalen Erwartungen zu berechnen.

Differenzengleichungen höherer als zweiter Ordnung erzeugen Mehrfachschwingungen, deren Analyse bedeutend komplexerer Natur ist, die aber dem charakteristischen Konjunkturbild der Realität kaum entsprechen.

Da die in Abbildung 1 sichtbare Kurvengestalt keinem eindeutig erkennbaren Bildungsgesetz $\mathrm{zu}$ folgen scheint, kann sie bereits nach wenigen Perioden auf verschiedene Art ,gestaltstreu'ergänzt werden. In den Abbildungen 2, 3 und 4 sind die von den Vpn einer Heidelberger Experimentserie am häufigsten gewählten Fortsetzungen eingezeichnet. Diese vermitteln einen groben Eindruck über die großen individuellen Unterschiede, die über das gesamte Experiment hinweg beobachtet wurden. Bemerkenswert ist auch, daß selbst in Perioden, in denen große Unsicherheit über die Fortsetzung der Reihe bestand, dennoch eine positive bzw. negative Veränderung der Reihe vorhergesagt wurde, obwohl bei rationaler Fehlerschätzung häufig auch der vorhergegangene Wert als Prognose hätte gewählt werden sollen, wenn an eine Minimierung des Prognosefehlers gedacht worden wäre. Die am häufigsten beobachteten Prognosewerte sind fast alle gewissermaßen ,gestaltstreu' aber kaum ,fehlerminimierend‘. Sogar die 6 Vpn, die - wie Abbildung 4 zeigt - den Wert 14 und die 5 Vpn, die den Wert 4 vorhergesagt haben, könnten ihre Prognosen durch lokale oder globale Gestaltsmerkmale rechtfertigen. 


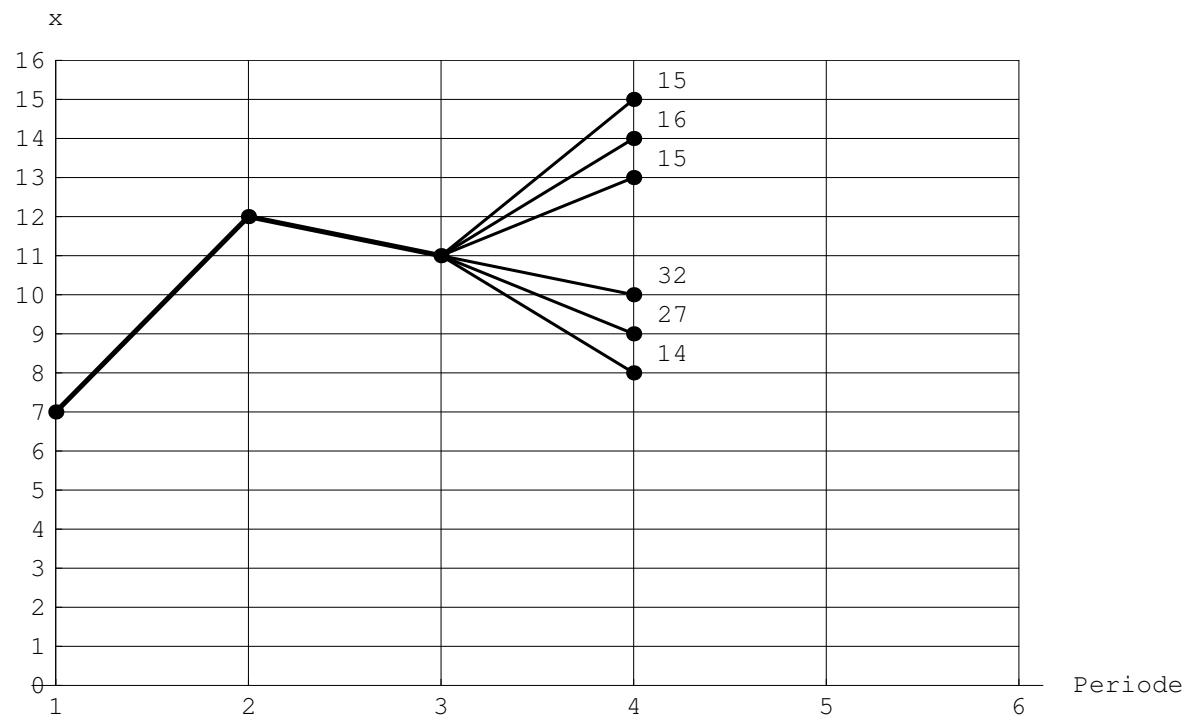

Abbildung 2: Prognosen für Periode 4.

Zwischen den individuellen Prognosen und den rationalen Erwartungen bestehen erhebliche Unterschiede. Da die Vpn im Experiment nicht die Möglichkeit haben, irgendwelche andere quantitative oder qualitative Prognoseverfahren anzuwenden, ist bei den einzelnen Vpn mit optimalen Prognosen im Sinne rationaler Erwartungen erfahrungsgemäß nicht zu rechnen.

Die individuellen Prognosen weichen erheblich von den rationalen Erwartungen ab. Dieses Ergebnis ist auch aus anderen vergleichbaren Experimenten bekannt. Es ist leicht zu erklären, denn die Versuchspersonen kennen die tatsächliche Erzeugungsgleichung der Zeitreihe nicht; sie sind auf visuelle Inferenz angewiesen und dürfen auch keine anderen Prognosetechniken anwenden.

Vergleicht man hingegen die durchschnittlichen Vorhersagen mit den rationalen Erwartungen, zeigt sich, wie in anderen Prognoseexperimenten, daß diese in zahlreichen Perioden wesentlich besser übereinstimmen, als dies bei den individuellen Prognosen der Fall ist. Aus rein statistischer Sicht dürfte dies in erster Linie auf das Phänomen des Fehlerausgleichs bei stochastisch unabhängigen Schätzungen zurückzuführen sein.

\section{Die Bounds \& Likelihood-Prozedur}

Zur Erklärung der im Experiment beobachteten mittleren Vorhersagen $f_{t+1}$ hat sich nach unserer Sicht ein Modell bewährt, das die erwartete Veränderung der Zeitreihe aus den beobachteten mittleren Veränderungen $b_{t}$ der Zeitreihe und der Umkehrwahrscheinlichkeit $l_{t}$ berechnet: In jeder Periode $t$ wird die Umkehrwahrscheinlichkeit $l_{t}$ geschätzt und die mittlere Veränderung im Fall des Abschwungs bzw. des Aufschwungs damit gewichtet. Dies führt zu den folgenden Gleichungen:

$$
\mathbf{f}_{\mathrm{t}+\mathbf{1}}=\mathrm{x}_{\mathrm{t}}+\mathbf{c}_{\mathrm{t}} \operatorname{sgn}\left(\mathrm{x}_{\mathrm{t}}-\mathrm{x}_{\mathrm{t}-\mathbf{1}}\right)
$$




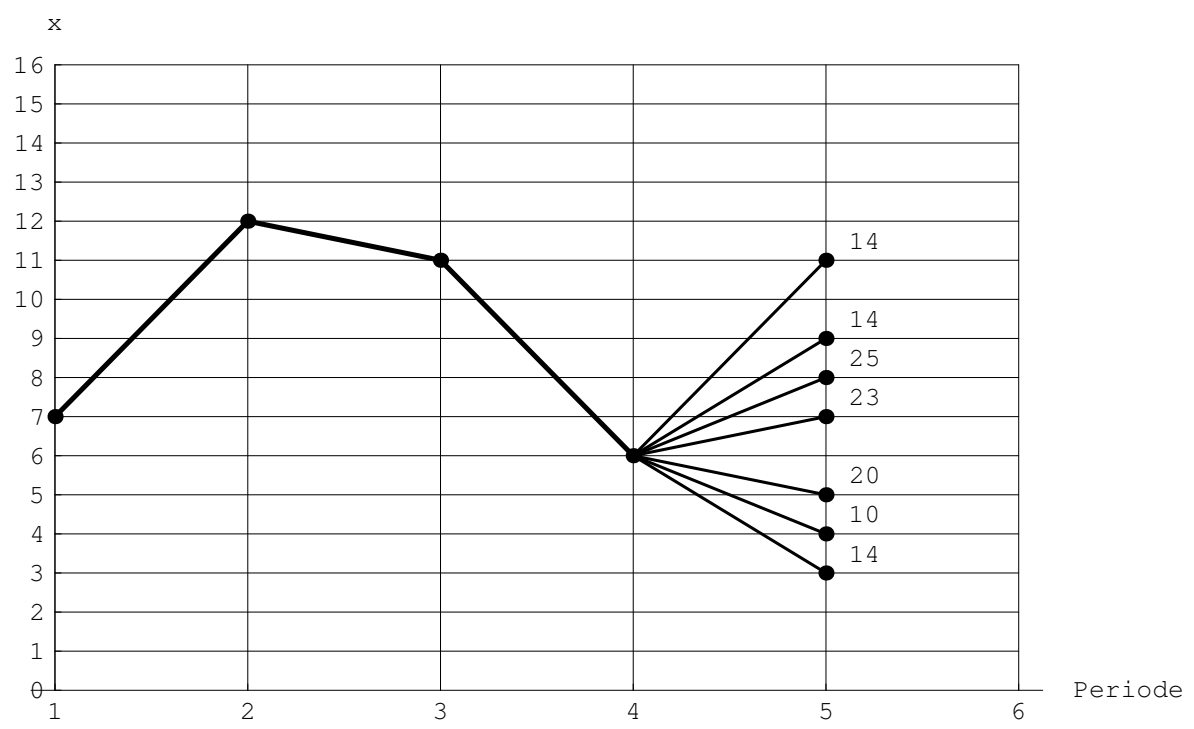

Abbildung 3: Prognosen für Periode 5.

mit den Koeffizienten

$$
c_{t}=b_{t}\left(1-2 l_{t}\right)
$$

und den zugehörigen Schätzwerten für die mittleren Veränderungen $b_{t}$

$$
b_{t}=\frac{1}{t-1} \sum_{j=2}^{t}\left|x_{j}-x_{j-1}\right|
$$

und für die Umkehrwahrscheinlichkeiten $l_{t}$

$$
l_{t}=\left\{\begin{array}{l}
\frac{1+\text { Anzahl der lokalen Maxima } \leq x_{t}}{2+\text { Anzahl der lokalen Maxima }} \text { für } x_{t}>x_{t-1} \\
\frac{1+\text { Anzahl der lokalen Minima } \geq x_{t}}{2+\text { Anzahl der lokalen Minima }} \text { für } x_{t}<x_{t-1} .
\end{array}\right.
$$

Der Erwartungswert $c_{t}$ ist nach oben wie nach unten durch die durchschnittlichen beobachteten Veränderungen begrenzt.

Die Größe $l_{t}$ schätzt im Falle eines Aufschwungs die relative Häufigkeit der lokalen Maxima oberhalb von $x_{t}$ zum Zeitpunkt $\mathrm{t}$ und im Falle eines Abschwungs die relative Häufigkeit der lokalen Minima unterhalb von $x_{t}$ zum Zeitpunkt t und somit die Umkehrwahrscheinlichkeit in beiden Richtungen ab. Offensichtlich wird mit $l=\frac{1}{2}$ gestartet, solange noch keine lokalen Extrema aufgetreten sind. Obere Umkehrpunkte sind als lokale Maxima und untere Umkehrpunkte als lokale Minima $\mathrm{zu}$ verstehen.

In Gleichung (5) sind ,Bayes-Schätzungen“ verwendet worden, die sich insbesondere in den Anfangsperioden, in denen noch wenige lokale Extrema zu sehen sind, als sinnvoll erweisen. Ist die Umkehrwahrscheinlichkeit $l_{t}$ gleich Null (Eins), so wird keine (eine) Umkehr vorhergesagt. Ist an der Stelle $x_{t}$ die Wahrscheinlichkeit einer 


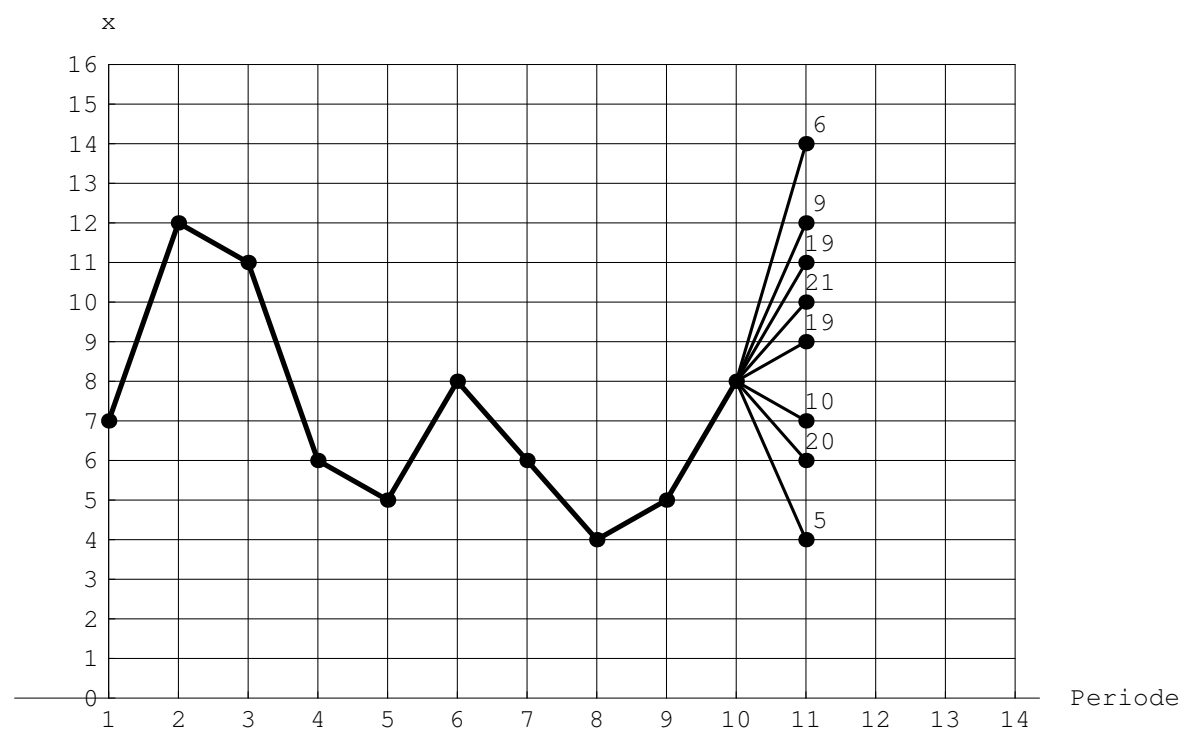

Abbildung 4: Prognosen für Periode 11.

Umkehr der bisherigen Änderungsrichtung gleich $1 / 2$, so kommt es zu der naiven Prognose $f_{t+1}=x_{t}$.

Da die Prognose der Änderung der Reihe durch den Betrag von $b_{t}$ (bound) begrenzt wird und von der Umkehrwahrscheinlichkeit (likelihood) abhängt, haben wir das mit den Gleichungen (2) bis (5) gegebene Modell als bounds \& likelihoodProzedur, kurz b\&l-Prozedur bezeichnet. Es handelt sich hierbei um eine rein apriorische Theorie, mit der durchschnittliche Vorhersagewerte ohne jegliche parametrische Anpassung an empirisch beobachteten Prognosen bestimmt werden können (Becker, Leopold and Kührer, 1997).

\section{Ergebnisse}

Das Experiment wurde bisher an den Universitäten in Heidelberg und Graz genau 333mal durchgeführt.

In der Abbildung 5 sind die Kollektiv-Vorhersagewerte den Vorhersagewerten der b\&l-Prozedur gegenübergestellt. Die Differenz zwischen den Kollektivprognosen und der b\&l-Prozedur ist durch den untersten Linienzug wiedergegeben und man kann die geringen Abweichungen voneinander ersehen.

Weiters bleibt die Frage, wie gut die b\&l-Prozedur die gegebene Zeitreihe vorhersagt. Der mittlere Prognosefehler, gemessen an den absoluten Abweichungen der b\&l-Prozedur zur tatsächlichen Zeitreihe, beträgt 1.934 Einheiten über die Perioden 7 - 42 gerechnet.

Die Werte der rationalen Erwartungshypothese (REH) zeigen für diese Perioden einen Prognosefehler von 1.833. Das Verhältnis dieser beiden Größen ergibt als Maß für die Effizienz $\eta$ der b\&l-Prozedur einen Wert $\eta=0.947$.

Dieses Effizienzmaß ändert sich nicht, falls alle Zufallsvariablen in ihrer Größe proportional zueinander verändert werden würden. Die b\&l-Prozedur ist eine li- 


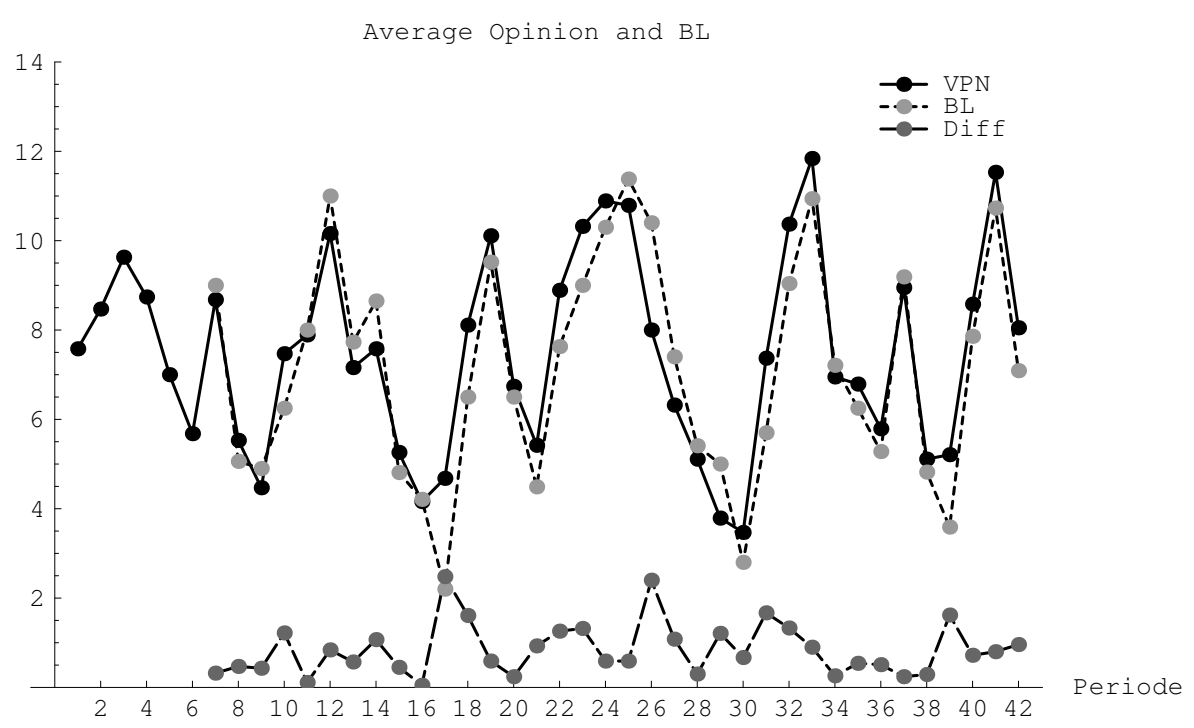

Abbildung 5: Vergleich(Diff) von durchschnittlichen Prognosen der Versuchspersonen (VPN) und Bounds\&Likelihood Prozedur (BL).

neare Prozedur. Wird die Systemvariable $x_{t}$ linear transformiert, z.B. durch eine Maßstabänderung in die neuen Variablen $z_{t}=k x_{t}$, so paßt sich die Prozedur sowohl in die Lage der Umkehrpunkte als auch im Ausmaß des Anstiegs an die neuen Variablen an. Dies hat zur Folge, daß sich auch die Prognosefehler um den Faktor $k$ ändern.

Da dies im übrigen auch für die rationalen Erwartungswerte der neuen Variablen gilt, folgt daraus, daß relative Fehlervergleiche gegenüber linearen Transformationen invariant sind.

Nun stellt sich die Frage, ob man die b\&l-Prozedur allgemein als normatives Verfahren verwenden kann. Hierzu wurde mittels Simulationsstudien untersucht, ob diese Prozedur auch bei Zeitreihen mit ähnlichem Zyklusmuster brauchbare Prognosen liefert. An Stelle der im Experiment verwendeten Daten wurden in einer Simulationsstudie 100.000 weitere Zeitreihen gemäß der Differenzengleichung (1) generiert und an diesen die b\&l-Prozedur getestet. Als ähnliche Zeitreihen wurden solche mit gleicher Dämpfung betrachtet, wobei der Zyklus im Bereich von 7 bis 12 Perioden variiert wurde. Als Ergebnis ergab sich eine mittlere Effizienz unserer b\&lProzedur im Gesamtdurchschnitt nahe bei 91\%. (Becker and Leopold-Wildburger, 1996, 1997), während sie im realen Experiment knapp unter 90\% gelegen ist. Die Zykluslänge hat in den betrachteten Intervallen von 7 bis 12 Perioden einen, wenn auch nur geringen Einfluß auf die Effizienz. Bemerkenswert ist die verhältnismäßig hohe Effizienz bereits innerhalb der ersten Perioden von 7 bis 15, die sich bis zu den Schlußperioden 34 bis 42 nur um wenig verbessert.

Aus den derzeit laufenden, erst teilweise abgeschlossenen Experimentserien mit Zusatzinformation für die Vpn in Form von Leitindikatoren zeigt sich bei erster Auswertung bereits, daß eine auf den vorliegenden Fall verallgemeinerte b\&l-Prozedur ebenfalls eine nennenswerte Effizienz aufweist. (Becker und Leopold-Wildburger, 
1999). Mit solchen zusätzlich gegebenen Informationen sollen komplexere, aber realistischere Problemsituationen analysiert werden.

\section{Literatur}

O. Becker. Experimentelle Untersuchung der Erwartungsbildung für eine Zeitreihe, in: Sauermann H. (ed.), Beiträge zur experimentellen Wirtschaftsforschung, Tübingen, 226-254, 1967.

O. Becker, and U. Leopold-Wildburger. The Bounds and Likelihood-Procedure A Simulation Study concerning the Efficiency of Visual Forecasting Techniques, in: Central European Journal of Operations Research and Economics, 4, 223-229, 1996.

O. Becker, and U. Leopold-Wildburger. The Advantage of the Bounds and Likelihood-Procedure - A Qualitative Forecasting Technique, in: Barcelo Jaime (ed.), Plenaries and Tutorials of the EUROXV - INFORMSXXXIV joint international meeting, Barcelona, 321-333, 1997.

O. Becker, and U. Leopold-Wildburger. Gestaltsgetreue Vorhersagen - Eine experimentelle Untersuchung mit zwei Informationsquellen, Working Paper der betriebswirtschaftlichen Institute der Sozial- und Wirtschaftswissenschaftlichen Fakultät der Karl-Franzens-Universität Graz, Mai 1999.

O. Becker, U. Leopold-Wildburger, and M. Kührer. A Qualitative Forecasting Approach, in: Rupnik V. (et al.), SOR'97 - Proceedings of the 4th International Symposium on Operational Research, Ljubljana, 1-12, 1997.

J.R. Hicks. Value and Capital, Clarendon Press, Oxford, 1939.

J.M. Keynes. The General Theory of Employment, Interest, and Money, Macmillan, London, 1936.

J.M. Keynes. The Collected Writings of John Maynard Keynes, vols 1 - 30, managing eds. A.Robinson and D.Moggridge, Macmillan, London, (1971-1988).

P. Ladreiter, and U. Leopold-Wildburger. Analysis of the Austrian Stock Market using Technical Trading Portfolios, Working Paper der Austrian Working Group for Banking and Finance Nr. 48, Wien, 1999.

U. Leopold-Wildburger. Some Selected Topics in Experimental Economics, in: International Transactions in Operational Research, 4, 165-174, 1997.

E. Lindahl. Studies in the Theory of Money and Capital, Allen and Unwin, London, 1939.

S. Makridakis, and S.C. Wheeelwright. The Handbood of Forecasting: A Manager's Guide, John Wiley, New York, 1987. 
J. Murphy. Visuelle Aktienanalyse, Campus Verlag, Frankfurt, 1997.

J.F. Muth. Rational Expectations and the Theory of Price Movements, Econometrica 29, 315-335, 1961.

J.A. Schumpeter. History of Economic Analysis, Oxford University Press, New York, 1954.

H. Thorton. Enquiry into the Nature and Effects of the Paper Currency of Great Britain, Macmillan, London, 1802.

Adressen der Autoren:

Professor Dr. Otwin Becker

Alfred Weber-Institut

Ruprecht-Karls-Universität Heidelberg

Grabengasse

D-69117 Heidelberg

Tel.: ++49 622761287

E-mail: O.becker.walldorf@t-online.de

Univ.-Prof. Mag. Dr. Ulrike Leopold-Wildburger

Institut für Statistik und Operations Research

Karl Franzens-Universität Graz

Universitätsstraße 15/E

A-8010 Graz

Tel.: ++433163803490

Fax: ++433163809560

E-mail: ulrike.leopold@kfunigraz.ac.at 